\title{
Pattern and associated factors of adherence to immunosuppressive medications in kidney transplant recipients at a referral center in Iran
}

This article was published in the following Dove Press journal: Patient Preference and Adherence

\author{
Omid Moradi \\ Iman Karimzadeh ${ }^{2}$ \\ Dorna Davani-Davari ${ }^{2}$ \\ Mojtaba Shafiekhani ${ }^{2}$ \\ Mohammad Mahdi Sagheb ${ }^{3}$ \\ 'Student Research Committee, Shiraz \\ University of Medical Sciences, Shiraz, \\ Iran; ${ }^{2}$ Department of Clinical Pharmacy, \\ Faculty of Pharmacy, Shiraz University of \\ Medical Sciences, Shiraz, Iran; \\ ${ }^{3}$ Nephrology-Urology Research Center \\ and Department of Internal Medicine, \\ Shiraz University of Medical Sciences, \\ Shiraz, Iran
}

Purpose: Ever increasing number of patients who receive kidney transplantation as a therapeutic option, approaches to increase graft survival as well as to identify factors that reduce the treatment outcomes should be taken into account. One of the heightened concerns after transplantation is non-adherence to immunosuppressive medications, which increases the risk of kidney failure or even rejection. The aim of this study was to evaluate factors associated with immunosuppressant medications adherence in kidney transplant recipients.

Patients and methods: Data were collected from 100 eligible kidney transplant patients referring to Shiraz Motahhari clinic and a private office of an attending nephrologist during 18 months. Adherence to immunosuppressive medications was assessed by Basel assessment of adherence to immunosuppressive medication scale at 2 time points.

Results: According to the findings, $55 \%$ of patients did not adhere to their posttransplantation immunosuppressive medications. The rate of adherence to immunosuppressive medications was not either statistically or clinically significant between time points zero and six months. None of the investigated factors including demographic, clinical and social-economical-cultural factors were significantly associated with patients' adherence to immunosuppressive regimen. Furthermore, there was no statistically significant association between immunosuppressive medication adherence and acute kidney rejection.

Conclusion: The rate of non-adherence to immunosuppressive medications was high. These data can be exploited by both physicians and policymakers to improve the rate of adherence to immunosuppressive medications amongst kidney transplant recipients.

Keywords: kidney transplantation, medication adherence, immunosuppressive agents

\section{Introduction}

Kidney transplantation is an appropriate and proven treatment for patients with endstage renal disease (ESRD). ${ }^{1}$ Hundreds of thousands of kidney transplantations are being performed annually around the world and the number of these operations are growing, and currently more than 180,000 patients with a functioning transplanted kidney are living in the United States. ${ }^{2}$

According to the statistics, 34,166 cases of kidney transplantations (4,436 grafts from cadaver and 29,730 grafts from living donor) took place in Iran upon till 2012. The annual rate of kidney transplantation in Iran ranks the highest among the Middle Eastern countries in 2011 and 2012. ${ }^{3}$ The kidney transplantation center in Shiraz, Southwest of Iran, is one of the most active renal transplantation centers in
Correspondence: Iman Karimzadeh Department of Clinical Pharmacy, Faculty of Pharmacy, Shiraz University of Medical Sciences, Karafarin street, $\mathrm{P} O$ Box 7|46864685, Shiraz, Iran

Tel +987132424128

Fax +987132424126

Email karimzadehiman@yahoo.com 
all of Iran. According to the literature, 1,200 cases of kidney transplantation had been performed from December 1988 to December 2003 in Shiraz. ${ }^{4}$

Kidney transplantation certainly has its own potential complications and side effects; hence, considerations should be taken into account. ${ }^{5}$ Patients' adherence to immunosuppressive medications plays a vital role in graft survival. ${ }^{6}$ Non-adherence to immunosuppressant medications is the third cause of graft loss after rejection and systemic infections. ${ }^{1}$ In a retrospective survey, Takemoto et al observed that by reducing the rate of adherence, the risk of graft loss was increased. ${ }^{7}$ Schweizer et al reported that $91 \%$ of the kidney transplant recipients are non-adherent to immunosuppressive medication, either lost their grafts or died after 6 to 12 months. ${ }^{8}$ Moreover, a review article implied that nonadherent recipients to immunosuppressive medications had sevenfold increase in the risk of rejection. ${ }^{9}$ It is estimated that non-adherence to immunosuppressive regimens costs 15-100 billion USD annually. ${ }^{10}$

Various factors affect the adherence rate among kidney transplant patients, such as age, gender, patient's attitudes toward immunosuppressive drugs, marital status, level of education, employment status, salary, the relationship between patients and health care personnel as well as the frequency of visiting doctor, side effects of medications, history of mental diseases, smoking, drug or alcohol addictions. ${ }^{11-14}$

The prevalence of non-adherence in kidney transplant recipients varies from $20 \%$ to more than $60 \% 15$ In this study, we attempted to evaluate the adherence rate of kidney transplant patients to their immunosuppressive medications and identifying the associated factors based on a self-reporting method in a referral center in Iran. To the best of our knowledge, there is only one published article regarding adherence to immunosuppressive medications at the setting of kidney transplantation amongst the Iranian population.

\section{Patients and methods}

\section{Study setting}

During an 18-month period from September 2015 to February 2017, this cross-sectional, observational study was conducted in the Mottahari specialty and subspecialty polyclinic affiliated to Shiraz University of Medical Sciences, Shiraz, Southwest of Iran and also a private office of one of the attending nephrologist. The institutional review board and medical ethics committee of the University approved the study protocols. Written informed consent form was taken from each patient in regard to data collection and taking part in the study or their responsible family members if they could not understand the study methods and goals or communicate properly with the interviewer due to being illiterate, having low socio-culture status, or having non-Persian languages (eg, Turkic, Arabic, or Luri).

\section{Patient selection}

Adult patients ( $\geq 18$ years of age) received kidney transplant and taking at least one immunosuppressive agent were included. Patients who had received kidney transplant previously (re-transplantation), had concomitant pancreatic or hepatic transplantation, or less than 6 months or more than 24 months after kidney transplantation were excluded from the study. This time period was considered in order to eliminate or minimize the possibility of recruiting patients whose non-adherence occurred during the early posttransplant period, when immunosuppressive regimen is changing or unstable. Non-adherence in this timeframe (6-24 months) might have the greatest clinical significance.

\section{Data collection}

Required clinical and demographic data were collected through face-to-face interview with the patients as well as reviewing their medical charts. For this purpose, a form was designed to record information on demographic factors (age and gender), social-economical-cultural factors (marital status, type of accommodation, education level, employment status, average monthly income, living condition, insurance type), habitual history (smoking, alcohol or drug addiction), and clinical factors related to their kidney transplantation (donor type, duration of kidney transplantation, cause of chronic kidney disease, type and duration of dialysis, underlying diseases as well as comorbidities, and acute rejection episodes), immunosuppressive regimen and other medications details (name, dose, frequency, starting date and duration of administration) were also collected.

\section{Adherence determination}

The patient's adherence level to immunosuppressive medication was determined by self-declaration and face-toface interviews via Basel assessment of adherence to immunosuppressive medication scale (BAASIS). This scale consists of four items about taking medication dimension, timing (drug holidays), dose reduction, and continuity of immunosuppressive medication administration. A positive answer to each of these items shows non- 
adherence to immunosuppressive medications. ${ }^{16}$ After answering the questions, the patients determined their own level of adherence, using the visual analog scale (VAS) in the range of 0 (not adherent) to 100 (complete adherence). The patient adherence level to immunosuppressive medication was determined in two phases of zero point and six months after the baseline, through faceto-face interviews or telephone calls. Causes of nonadherence to immunosuppressive medications were also recorded. Patients' beliefs as well as motivations about the immunosuppressive therapy and using auxiliary devices to remember immunosuppressive medications taking time (eg, timed pillboxes, calendar) were collected.

\section{Statistical analyses}

Categorical data were reported as a percentage and continuous variables were expressed as either means \pm standard deviations (SD) or median (interquartile range [IQR]). Chisquare test or Fisher's exact test was performed (if more than $25 \%$ of the categories had frequencies below five) to compare the rate of adherence between two time points as well as the possible association of immunosuppressive adherence with acute rejection episodes. Logistic regression analysis with odds ratio (OR) and $95 \%$ confidence interval (CI) via a stepwise method was performed to determine the possible association with different demographic, socialeconomical-cultural, habitual, and clinical factors as well as development of acute rejection episodes. Variables with $P$-values less than 0.3 were selected for the final multivariate logistic regression model. $P$-values less than 0.05 were considered to be statistically significant. All the above analyses were carried out by the Statistical Package for the Social Sciences (SPSS) version 20 software (IBM company, New York, NY, United States).

\section{Results}

Initially, a total of 110 patients were screened. Ten patients were excluded due to re-transplantation or having concurrent pancreatic or liver transplantation. Finally, 100 patients were included. Table 1 summarizes the demographic, clinical, and social-economical-cultural data of the studied population. The age of patients ranged between 22 and 72 years. Sixty eight percent of the cohort was men. Hypertension and diabetes were the two major causes of ESRD. Most of them (84\%) were under hemodialysis before kidney transplantation. More than onefourth (27\%) were currently unemployed. About one-fifth (19\%) had an academic education. Most participants
Table I Demographic, clinical, and social-economical-cultural properties of the study population $(n=100)$

\begin{tabular}{|c|c|}
\hline \multirow{3}{*}{$\begin{array}{l}\text { Gender (\%) } \\
\text { Male } \\
\text { Female }\end{array}$} & \multirow{3}{*}{$\begin{array}{l}68 \\
32\end{array}$} \\
\hline & \\
\hline & \\
\hline Age, Mean \pm SD (years) & $43 \pm 14$ \\
\hline \multicolumn{2}{|l|}{ Donor type (\%) } \\
\hline Non-relative living donor & 7 \\
\hline Relative living donor & 2 \\
\hline Deceased & 91 \\
\hline \multicolumn{2}{|l|}{ Cause of chronic kidney disease (\%) } \\
\hline Hypertension & 27 \\
\hline Diabetes mellitus & 19 \\
\hline Others ${ }^{i}$ & 54 \\
\hline \multicolumn{2}{|l|}{ Underlying disease (\%) } \\
\hline Hypertension & 31 \\
\hline Diabetic mellitus & 18 \\
\hline Others ${ }^{\mathrm{ii}}$ & 51 \\
\hline \multicolumn{2}{|l|}{ Dialysis type (\%) } \\
\hline Hemodialysis & 84 \\
\hline Peritoneal & 13 \\
\hline Never had dialysis & 3 \\
\hline Post-transplantation duration, Mean \pm SD (months) & $\begin{array}{l}14.37 \\
\pm 5.44\end{array}$ \\
\hline $\begin{array}{l}\text { Number of consumed medications for each patient, } \\
\text { Median (IQR) }\end{array}$ & $6(6.75)$ \\
\hline \multicolumn{2}{|l|}{ Employment status (\%) } \\
\hline Not able to work & 30 \\
\hline Unemployed & 27 \\
\hline Employed & 29 \\
\hline Retired & 16 \\
\hline \multicolumn{2}{|l|}{ Insurance status (\%) } \\
\hline Insured & 99 \\
\hline Uninsured & 1 \\
\hline \multicolumn{2}{|l|}{ Educational years (\%) } \\
\hline Illiterate & 9 \\
\hline 6 years $^{\mathrm{iii}}$ & 72 \\
\hline More than 12 years $^{\mathrm{iv}}$ & 19 \\
\hline \multicolumn{2}{|l|}{ Marital status (\%) } \\
\hline Married & 73 \\
\hline Single & 25 \\
\hline Divorced & 2 \\
\hline \multicolumn{2}{|l|}{ Opioid addiction (\%) } \\
\hline Never & 89 \\
\hline Previously & 9 \\
\hline Currently & 2 \\
\hline
\end{tabular}

(Continued) 
Table I (Continued).

\begin{tabular}{|l|l|}
\hline Alcohol consumption (\%) & 90 \\
Never & 10 \\
Previously & 0 \\
Currently & \\
\hline Cigarette smoking (\%) & 83 \\
Never & 15 \\
Previously & 2 \\
Currently & \\
\hline Monthly income (USD) (\%) & 18 \\
Less than 60 & 12 \\
$60-150$ & 70 \\
More than I50 & 712 \\
\hline
\end{tabular}

Notes: 'Nephrolithiasis, Glomerulonephritis, Polycystic kidney disease. ii Dyslipidemia, Hypothyroidism, Depression. iiischool grade. ${ }^{\text {iv }}$ Academic grade.

(96\%) lived with their families. Near three-fourth $(73 \%)$ of patients were married or engaged. Ten percent and $9 \%$ of the patients were alcohol and opium addicts, respectively, either before or after transplantation. Fifteen percent of patients quit cigarette after transplantation and currently $2 \%$ of them were cigarette smokers. None of the patients had a history of psychiatric disorders. The median number of medications used by patients was six. Seventy percent of patients had monthly income above \$150 USD.

At the time points zero and six months, $59 \%$ and $54 \%$ of the patients were adherent to their immunosuppressive medications. There was no significant difference in the rate of adherence in the two stages of study ( $P$-value $>0.05$ ). Overall, $55 \%$ of the patients did not adhere to their immunosuppressive medications at least in one of the studied time points. The mean \pm SD patients' self-scoring about their adherence to the immunosuppressive medication by VAS was $92 \pm 11$. It ranges from 50 to 100 .

The main reasons for non-adherence to immunosuppressive regimen were forgetfulness $(83 \%)$, financial problems (6\%), and unavailability of medications (3\%). Adverse drug reactions and allergies were responsible for non-adherence in two patients.

Most (98\%) of the patients declared that they had high motivation for transplantation and initiation of their immunosuppressive therapy before transplantation. At the time of interview, the rate of patients' motivation to continue immunosuppressive treatment after kidney transplant was $99 \%$. Half $(50 \%)$ of them were reminded by their family members, especially their wives. The use of reminding devices was reported in $60 \%$ of the patients, and the alarm clock was the most common one.
Table 2 shows the possible association between different demographic, clinical, and social-economical-cultural of the study population and adherence to immunosuppressive medications. According to univariate logistic regression analysis, duration of immunosuppression, type of donor, type of accommodation, cigarette smoking, and opium addiction were selected. However, there was no significant association between these variables and adherence based on the multivariate logistic regression model.

Only $9 \%$ of the patients experienced acute rejection episodes, of which 5 and 4 were in the category of adherent and non-adherent patients, respectively. There was no significant association between patients' adherence to immunosuppressive medications and episode of acute rejection.

\section{Discussion}

In the current survey, $55 \%$ of the patients did not adhere to their immunosuppressive medications. The rate of nonadherence in the setting of kidney transplantation was reported from $5 \%$ to more than $50 \% .{ }^{17,18}$ This variation can be due to several reasons, such as difference in the definition as well as detection method of patient's adherence and the lack of similarity between various studies regarding their methodology, population, and risk of nonadherence. For example, in a study by Didlake et al, ${ }^{19}$ patients with a serum cyclosporine level below $25 \mathrm{ng} / \mathrm{mL}$ were classified as "major clinical non-compliant", and those with a serum cyclosporine level greater than $25 \mathrm{ng} / \mathrm{mL}$ were defined as "minor clinical non-compliant". In another study on 105 kidney transplant patients, weight gain greater than $20 \%$, cyclosporine whole blood levels less than $30 \mathrm{ng} / \mathrm{ml}$, or missing more than $20 \%$ of their clinical visits were considered as non-adherence. ${ }^{20}$ On the other hand, some studies used the pill counting method to assess non-adherence, while in another study, it was determined via sending a questionnaire through emails to kidney transplant recipients. The only relevant study done in Iran was conducted by Ghods and Nasrollahzadeh on 286 kidney transplant patients during 3 months from February to April 2002 in the Hasheminejad University hospital in Tehran. In this study, non-adherence level to immunosuppressive medications was reported to be $25.5 \%,{ }^{1}$ which was much less than ours $(55 \%)$. This difference in the results can be due to several reasons. First, patients who did not take three or more non-sequential doses of immunosuppressive drugs in one month were considered as "minor non- 


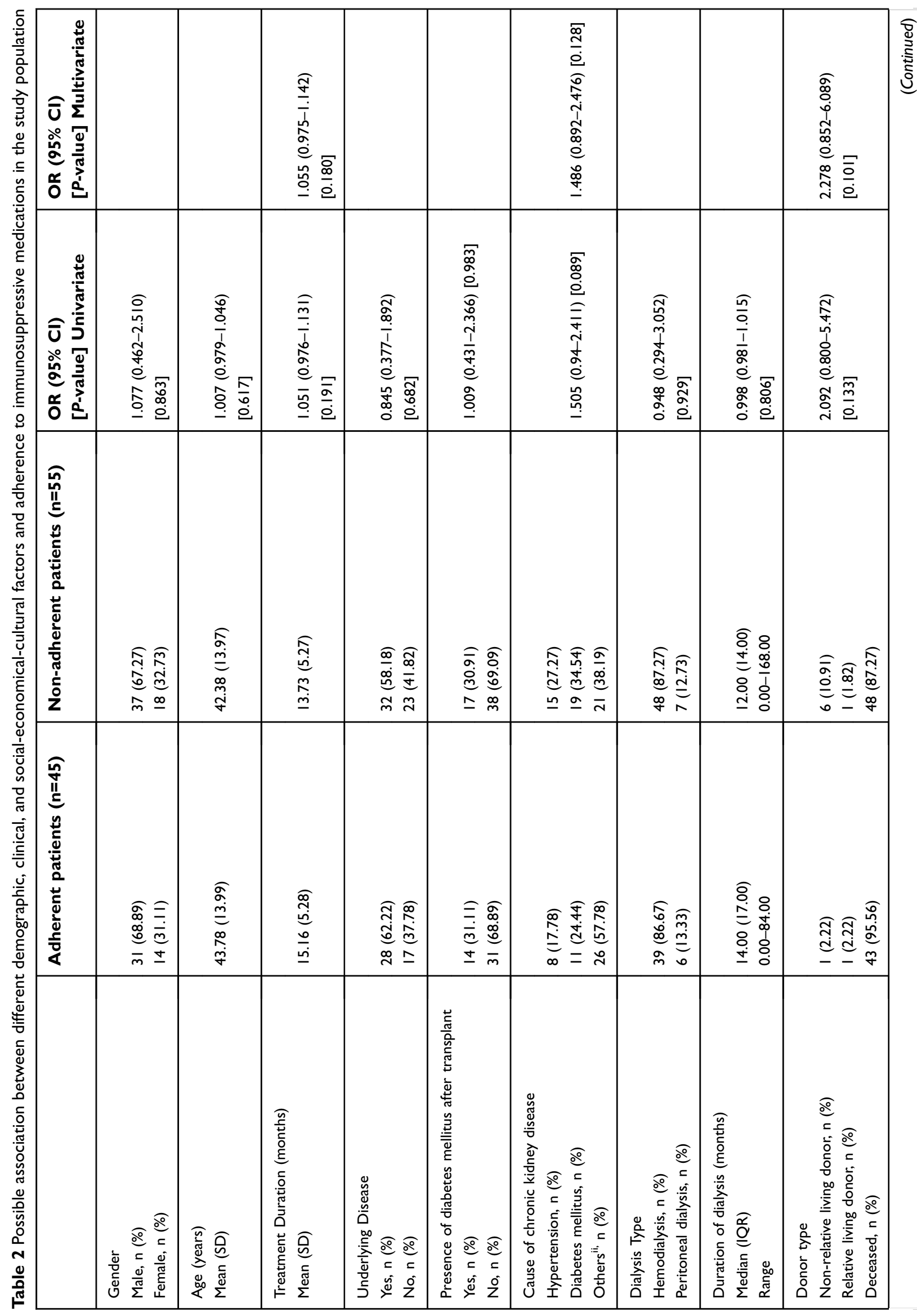




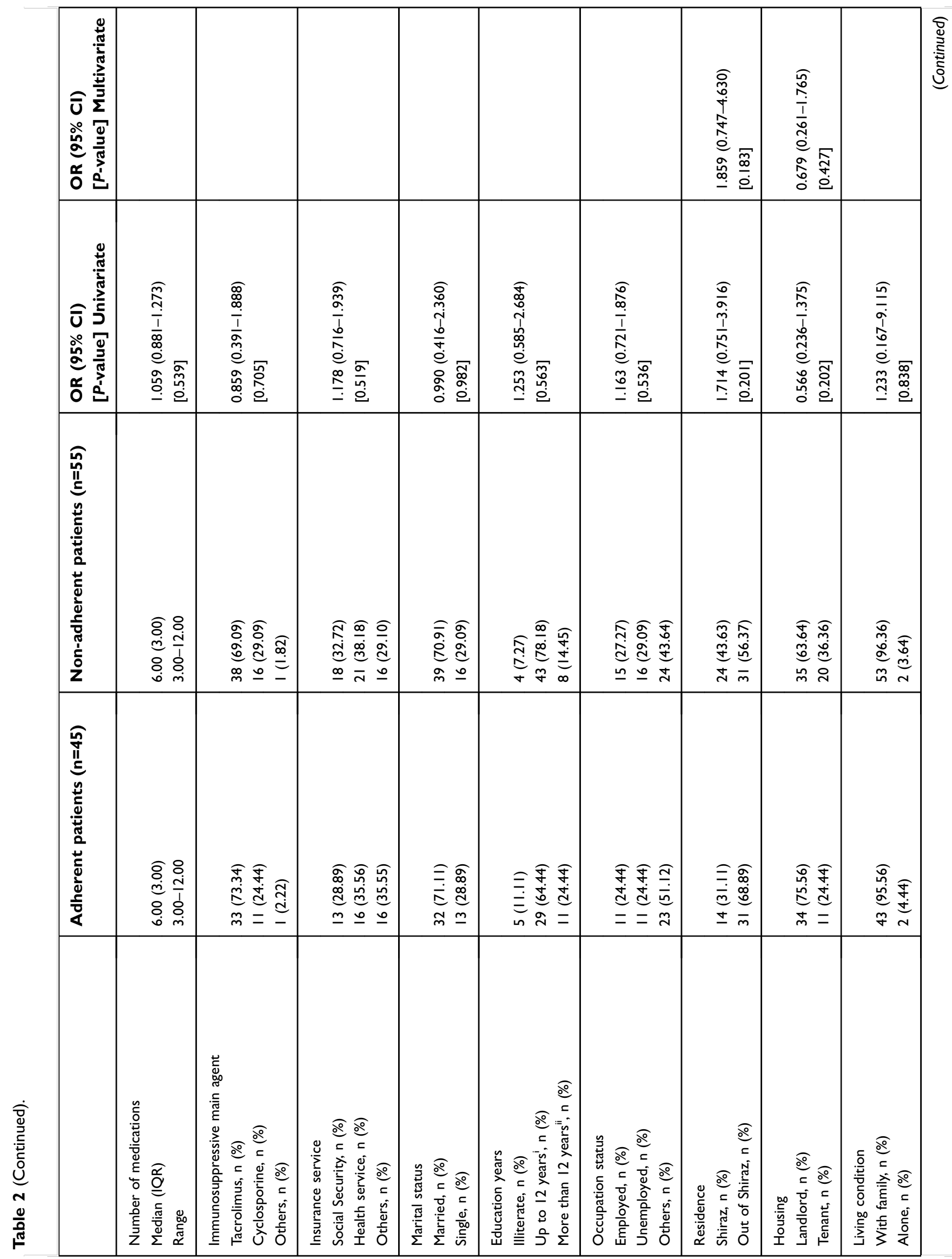




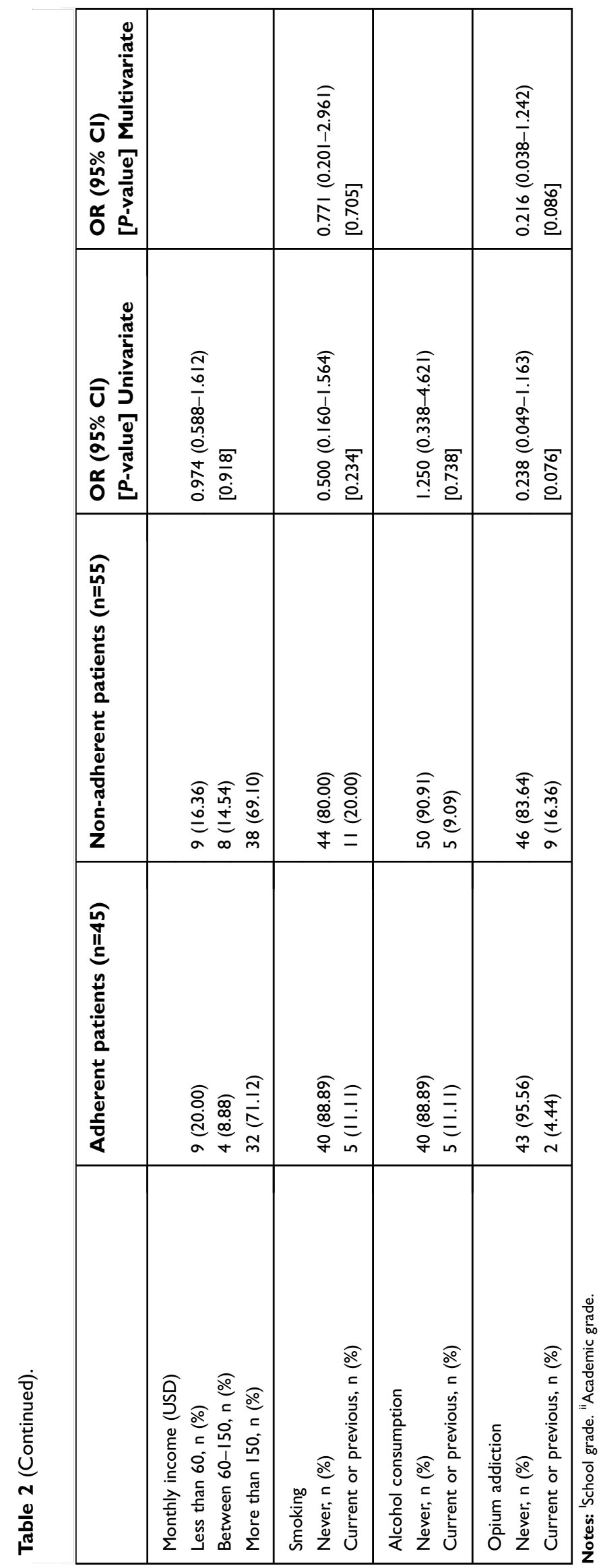


compliance" and those who stopped three or more consecutive doses per month as well as those who stopped taking immunosuppressive drugs for several days, weeks, or months were defined as "major non-compliance" in their study. ${ }^{1}$ While as mentioned in the method section, we used the BAASIS questionnaire to assess our patients' adherence. According to this questionnaire, the definition of nonadherence appears to be more robust, and all aspects of time, dose, as well as continuity of taking immunosuppressive medications were evaluated simultaneously. Secondly, in our study, we followed patients' adherence in two phases, time zero and six months later and patients who were nonadherent at each of these two phases were considered as non-adherent. However, this approach was not considered in the Ghods and Nasrollahzadeh investigation. ${ }^{1}$

Two systematic reviews specifically identified and discussed factors relevant to medication non-adherence, explicitly in kidney transplant patients. ${ }^{21,22}$ It seems that younger kidney recipients might underestimate the effects of their immunosuppressant drugs. Also, physical as well as cosmetic adverse effects of immunosuppressive drugs, such as acne, hair loss, gingival hyperplasia, obesity, and hirsutism can be more intolerable for young people. In almost all previous studies, younger patients had a lower level of adherence than older ones. However, in the current study, there was no significant association between age and non-adherence $(P$-value $>0.05)$. This might be related to the distribution of age in our cohort. In this regard, $67 \%$ of our patients were over 35 years old and those less than 25 years of age consisted only $11 \%$ of the population.

In previous studies, it was shown that by increasing the post-transplantation duration, the level of adherence decreases. ${ }^{1,23}$ One of the plausible reasons for this phenomenon is that by increasing this duration, the patient's positive opinion about the benefits of immunosuppressive drugs might be reduced. Ravi et al reported that adherence to (pegylated) interferon alpha and ribavirin alone or their combination diminished significantly over the treatment course in Iranian patients with chronic hepatitis $C^{24}$ However, in our study, no significant association was found in this regard. In addition, although the rate of adherence decreased by $5 \%$ after 6 months compared to the baseline, this difference was neither statistically nor clinically significant. One of the reasons that can affect the association between the non-adherence level and kidney post-transplant duration in our study can be the time points selected for the assessment of non-adherence. In the present study, recruited patients were between 6 and 24 months after transplantation. Apart from low sample size, the short duration of the study, as well as the close time intervals and follow-up between date of transplantation and determining patient's adherence might justify these findings.

A number of studies have shown that poverty and poor socio-economic levels are one of the factors that contribute to the adherence of patients with immunosuppressive regimens in kidney transplant recipients, but in our study, there was no significant association between the average monthly income and their adherence rate $(P$-value $>0.05)$. One of the reasons for this finding is that in Iran, as a developing country, the government pays all the costs of kidney transplant recipients. The main immunosuppressive drugs, including calcineurin inhibitors (cyclosporine and tacrolimus) and antimetabolites (mycophenolate and azathioprine), are available as generic types in our country, which are much cheaper than the original brands. On the other hand, insurance services or charity organizations cover all the costs for kidney transplant recipients, and patients pay only small fraction of the amount for drugs. Therefore, almost all patients have the ability to pay for their immunosuppressive medications.

Presence of underlying disease as whole and specifically diabetes along with diseases causing chronic kidney disease (eg, hypertension, diabetes) had no significant association with adherence to immunosuppressive regimen in our cohort. In contrast to these data, Jindal et al reported that comorbid conditions (ischemic heart failure) as well as causes of ESRD (hypertension and diabetes) significantly associated with non-adherence in recipients of kidney transplants in the US. The authors did not provide any explanation or rational for these findings. ${ }^{25}$ Furtherer studies might be warranted to prove this issue.

Forgetfulness as the most prevalent reason was declared by the participants for non-adherence in our study. This was also mentioned in other similar studies. In a study on 218 kidney transplant recipients, it was reported that $62.4 \%$ of the patients were non-adherent to their immunosuppressive medications due to forgetfulness. ${ }^{26}$ Interestingly, forgetfulness is one of the major reasons for non-adherence to medications in other clinical settings in Iran such as HIV ${ }^{27}$ In general, financial problems and unavailability of medications are the other two factors related to patients' non-adherence. This is related to the fact that at some time within the study period, due to lack of prominent generic type of mycophenolate (Roche, Cellcept ${ }^{\circledR}$, Basel, Switzerland), many patients had to purchase other brands of this medication 
(eg, Clausen, Suprimune $\AA$, Montevideo, Uruguay; Daropakhsh, Cytocept ${ }^{\circledR}$, Tehran, Iran; Osve, Citogan ${ }^{\circledR}$, Tehran, Iran) that were not covered by the insurance companies and might not be freely available to most of the patients. The same story goes for Prograf $\AA$, a well-known brand name of tacrolimus.

Non-adherent patients are expected to experience more acute rejection episodes. In a study by Schweizer et al, $91 \%$ of the non-adherent patients lost their grafts or died within 6-12 months after transplantation. ${ }^{8}$ Similarly, the incidence of acute rejection in non-adherent patients were 4.8 times more than those adherent with their immunosuppressive regimen in Iran $(P<0.001) .{ }^{1}$ However, in our study, the rate of acute rejection episodes was comparable between adherent and non-adherent patients. The incomplete medical record of patients (especially in the private office), as well as the low sample size can justify this finding. In other words, the actual number of patients experiencing at least one episode of acute rejection might be more than what we identified. In this regards, Salahi et al reported that among 1,200 consecutive kidney transplant patients at Shiraz Organ Transplant Center during a 15-year period from December 1988 to December 2003, the rate of graft rejection in alive and dead renal recipients was $6.1 \%$ and $41.7 \%$, respectively. ${ }^{28}$

Strengths of the current study included measuring and determining the level of immunosuppressive medication adherence, using a well-known and valid scale (BAASIS) at two time points for each participant. However, this study has some limitations. First, the sample size was relatively small and the possibility of second type error in statistical analyses cannot be ignored; thus, relevant results should be interpreted with caution. Second, determining adherence was based on a self-reporting method, which merely depends on patients' report and more precise and objective methods to determine the level of adherence such as electronic equipment or pill counting methods were not taken into account due to lack of patients' cooperation as well as required facilities for its implementation. Third, this study only considered acute rejection as clinical outcome and other clinical outcome indexes that can be relevant to patient adherence such as long-term survival of kidney graft and overall survival rate of patients were not measured. Fourth, only patients who referred to the clinic or private office were evaluated, and other individuals who had lost their transplanted organ or died were not evaluated. It is advisable that all transplant recipients, both patients who have a healthy and active transplanted kidney and those who have lost their allograft, should be monitored.

\section{Conclusion}

According to results of this study, more than half (55\%) of kidney transplant recipients were non-adherent to their immunosuppressive regimen in at least one of the time points based on a self-reporting method (BAASIS). The rate of adherence to immunosuppressive medications was not either statistically or clinically significant between time points zero and six months. There was no significant association between various studied demographic, clinical, as well as social-cultural-economical factors and adherence to immunosuppressive medications. Forgetfulness was the biggest reason declared by the participants for their non-adherence. Episodes of acute rejection were comparable between adherent and non-adherent subjects. The data can be exploited by both physicians and policymakers to improve the rate of adherence to immunosuppressive medications and remove barriers of nonadherence in kidney transplantation.

\section{Acknowledgments}

The authors wish to thank Mr. H. Argasi at the Research Consultation Center (RCC) of Shiraz University of Medical Sciences for his invaluable assistance in editing this manuscript. The present article was extracted from the data of PharmD thesis of Omid Moradi. It was financially supported by the Vice-Chancellery of Research and Technology of Shiraz University of Medical Sciences with the grant number: 95-01-05-11773.

\section{Disclosure}

The authors report no conflicts of interest in this work.

\section{References}

1. Ghods AJ, Nasrollahzadeh D. Noncompliance with immunnosuppressive medications after renal transplantation. Exp Clin Transplant. 2003;1(1):39-47.

2. Kasper D, Fauci A, Hauser S, Longo D, Jameson J, Loscalzo J, eds. Harrison's Principles of Internal Medicine. 19th ed. New York: McGraw-Hill; 2015:19e.

3. Ghods AJ. The history of organ donation and transplantation in Iran. Exp Clin Transplant. 2014;12:38-41.

4. Malek-Hosseini S, Razmkon A, Mehdizadeh A, et al. Long-term results of renal transplantation: a single-center analysis of 1200 transplants. Transplant Proc. 2006;38(2):454-456.

5. Monahan FD, Sands JK, Neighbors M, Marek JF, Green CJ. Phipps ' Medical-Surgical Nursing: Health and Illness Perspectives: Europe, Middle East and African Edition. 8th ed. St. Louise, MO: Elsevier Mosby; 2007. 
6. Desmyttere A, Dobbels F, Cleemput I, De Geest S. Noncompliance with immunosuppressive regimen in organ transplantation: is it worth worrying about? Acta Gastroenterol Belg. 2005;68(3):347.

7. Takemoto S, Pinsky B, Schnitzler M, et al. A retrospective analysis of immunosuppression compliance, dose reduction and discontinuation in kidney transplant recipients. Am J Transplant. 2007;7 (12):2704-2711. doi:10.1111/j.1600-6143.2007.01966.x

8. Schweizer RT, Rovelli M, Palmeri D, Vossler E, Hull D, Bartus S. Noncompliance in organ transplant recipients. Transplantation. 1990;49 (2):374-376

9. Laederach-Hofmann K, Bunzel B. Noncompliance in organ transplant recipients: a literature review. Gen Hosp Psychiatry. 2000;22(6):412-424.

10. Gorevski E, Succop P, Sachdeva J, et al. Is there an association between immunosuppressant therapy medication adherence and depression, quality of life, and personality traits in the kidney and liver transplant population? Patient Prefer Adherence. 2013;7:301.

11. Ghods A, Nasrollahzadeh D, Argani H. Risk factors for noncompliance to immunosuppressive medications in renal transplant recipients. Transplant Proc. 2003;35(7):2609-2611.

12. Brahm M, Manfro R, Mello D, Cioato S, Gonçalves L. Evaluation of adherence to immunosuppressive drugs in kidney transplantation by control of medication dispensing. Transplant Proc. 2012;44(8):23912393. doi:10.1016/j.transproceed.2012.08.001

13. Bunzel B, Laederach-Hofmann K. Solid organ transplantation: are there predictors for posttransplant noncompliance? A literature overview. Transplantation. 2000;70(5):711-716.

14. Stolyar A, Tomilina N. Impact of smoking on kidney transplantation outcomes. Ter Arkh. 2016;88(12):45-50. doi:10.17116/terarkh2016881245-50

15. Chisholm-Burns MA, Spivey CA, Sredzinski E, Butler SL. Intervention toolbox to promote immunosuppressant therapy adherence in adult renal transplant recipients. J Am Pharm Assoc. 2012;52 (6):816-822. doi:10.1331/JAPhA.2012.11083

16. Dobbels F, Berben L, De Geest S, et al. The psychometric properties and practicability of self-report instruments to identify medication nonadherence in adult transplant patients: a systematic review. Transplantation. 2010;90(2):205-219. doi:10.1097/TP.0b013e3181e346cd

17. Leendertse AJ, Egberts AC, Stoker LJ, van Den Bemt PM. Frequency of and risk factors for preventable medication-related hospital admissions in the Netherlands. Arch Intern Med. 2008;168(17):1890-1896. doi:10.1001/archinternmed.2008.3
18. Azmandian J, Sohrevardi SM, Fazeli F, et al. Diltiazem co-treatment in renal transplant patients receiving cyclosporine with respect to concentration at two hours (C2). Iran J Pharm Sci. 2011;7(1):3-6.

19. Didlake R, Dreyfus K, Kerman R, Van Buren C, Kahan B. Patient noncompliance: a major cause of late graft failure in cyclosporine-treated renal transplants. Transplant Proc. 1988;20(3 Suppl 3):63-69.

20. Kiley DJ, Lam CS, Pollak R. A study of treatment compliance following kidney transplantation. Transplantation. 1993;55(1):51-56.

21. Rebafka A. Medication adherence after renal transplantation-a review of the literature. J Ren Care. 2016;42(4):239-256. doi:10.1111/ jorc. 12181

22. Belaiche S, Décaudin B, Dharancy S, Noel C, Odou P, Hazzan MJ. Factors relevant to medication non-adherence in kidney transplant: a systematic review. Int J Clin Pharm. 2017;39(3):582-593. doi:10.1007/s11096-017-0436-4

23. Sketris I, Waite N, Grobler K, West M, Gerus S. Factors affecting compliance with cyclosporine in adult renal transplant patients. Transplant Proc. 1994;26(5):2538-2541.

24. Ravi S, Nasiri Toosi M, Karimzadeh I, Ahadi-Barzoki M, Khalili H. Adherence to chronic hepatitis $\mathrm{C}$ treatment regimen: first report from a referral center in iran. Hepat Mon. 2013;13(6):e11038-e11038. doi:10.5812/hepatmon

25. Jindal R, Neff R, Abbott K, et al. Association between depression and nonadherence in recipients of kidney transplants: analysis of the United States renal data system. Transplant Proc. 2009;41(9):36623666. doi: 10.1016/j.transproceed.2009.06.187

26. Griva K, Davenport A, Harrison M, Newman SP. Non-adherence to immunosuppressive medications in kidney transplantation: intent vs. forgetfulness and clinical markers of medication intake. Ann Behav Med. 2012;44(1):85-93. doi:10.1007/s12160012-9359-4

27. Khalili H, Rohani R, Seyedalinaghi S, Hajiabdolbaghi M, DashtiKhavidaki S, Talasaz AH. Adherence to antiretroviral therapy among Iranian HIV/AIDS patients. Curr Clin Pharmacol. 2012;7 (2):111-115.

28. Salahi H, Jalaeian H, Nikeghbalian S, et al. The comparison of clinical and biochemical parameters among 1200 alive and dead renal transplant recipients. Saudi J Kidney Dis Transplant. 2007;18 (3):439-442.

\section{Publish your work in this journal}

Patient Preference and Adherence is an international, peer-reviewed, open access journal that focusing on the growing importance of patient preference and adherence throughout the therapeutic continuum. Patient satisfaction, acceptability, quality of life, compliance, persistence and their role in developing new therapeutic modalities and compounds to optimize clinical outcomes for existing disease states are major areas of interest for the journal. This journal has been accepted for indexing on PubMed Central. The manuscript management system is completely online and includes a very quick and fair peer-review system, which is all easy to use. Visit http:// www.dovepress.com/testimonials.php to read real quotes from published authors. 